\section{Tradução e adaptação transcultural da Multiple Sclerosis Walking Scale - 12 (MSWS-12) para a língua portuguesa do Brasil}

\author{
Translation and cross-cultural adaptation of the \\ Multiple Sclerosis Walking Scale (MSWS-12) \\ into Brazilian Portuguese
}

\author{
1 Programa de Pós-graduação \\ em Neurologia, Universidade \\ Federal do Estado do Rio de \\ Janeiro, Rio de Janeiro, Brasil. \\ 2 Hospital Universitário \\ Gaffrée e Guinle, \\ Universidade Federal do \\ Estado do Rio de Janeiro, \\ Rio de Janeiro, Brasil. \\ 3 Instituto Nacional de \\ Câncer, Rio de Janeiro, Brasil. \\ Correspondência \\ L. C. S. Thuler \\ Departamento de Neurologia, \\ Hospital Universitário \\ Gaffrée e Guinle, \\ Universidade Federal do \\ Estado do Rio de Janeiro. \\ Rua Mariz e Barros 775, \\ Rio de Janeiro, $R J$ \\ 20270-004, Brasil. \\ lthuler@inca.gov.br
}

\begin{abstract}
Poor walking performance is predictive of heart disease and osteoporosis and increases the risk of death in the elderly. Gait and vision have been identified as the most valuable physical functions according to multiple sclerosis patients' perceptions. The objective of this study was to perform a translation and cross-cultural adaptation of the Multiple Sclerosis Walking Scale (MSWS-12) into Brazilian Portuguese. A study of cross-cultural adaptation was conducted in ten steps. Participation in the study included four translators, two back-translators, twelve medical experts, twelve patients, twelve healthy subjects, and a Portuguese language expert. Only the question "Did standing make it more difficult to do things?" posed difficulty in the translation process. Maximum time for completion was less than three minutes (171 seconds). Internal consistency analyses showed high reliability (Cronbach's alpha $=$ 0.94). The content validation and internal consistency stages were completed satisfactorily.
\end{abstract}

Gait; Multiple Sclerosis; Translating; Questionnaires
Leandro Alberto Calazans Nogueira 1

Carolinne Baitelli 2

Regina Maria Papais Alvarenga 1

Luiz Claudio Santos Thuler 2,3

A capacidade de caminhar tem importantes implicações para a saúde de um indivíduo. O baixo desempenho da caminhada é preditivo de doença cardíaca e aumenta a probabilidade de morte em idosos 1 . Pessoas que são reabilitadas frequentemente dizem que a marcha é o objetivo mais importante de sua recuperação 2 .

A marcha influencia negativamente a qualidade de vida de pacientes com esclerose múltipla 3 e juntamente com a visão são as funções corporais mais valiosas na percepção dos pacientes 4 . Embora alguns instrumentos de avaliação de mobilidade já tenham sido traduzidos para a língua portuguesa, nenhum é direcionado para avaliação da marcha na esclerose múltipla. A Multiple Sclerosis Walking Scale - 12 (MSWS12) é um questionário autoaplicável que mede o impacto da esclerose múltipla na caminhada. Os valores variam entre 0 e 80 , e valores mais altos indicam pior desempenho ou maior dificuldade para caminhar. Altos valores de reprodutibilidade, confiabilidade e validade dessa escala têm sido documentados 5,6. O objetivo do presente estudo foi traduzir e realizar a adaptação transcultural do conteúdo da MSWS-12 para a língua portuguesa do Brasil.

\section{Metodologia}

Um estudo de tradução e adaptação transcultural foi conduzido seguindo as recomendações de 
Guillemin et al. ${ }^{7}$, Beaton et al. ${ }^{8}$ e Wild et al. ${ }^{9}$. O projeto de pesquisa foi aprovado no comitê de ética e pesquisa do Hospital Universitário Gaffrée e Guinle (HUGG).

A MSWS-12 original foi traduzida do inglês para o português por dois tradutores nativos na língua inglesa (com fluência na língua portuguesa e com experiência profissional em tradução) e dois nativos na língua portuguesa (com formação acadêmica e experiência profissional em tradução). O instrumento reconciliado foi enviado a dois retrotradutores independentes, nativos na língua inglesa com fluência na língua portuguesa, que não haviam participado da etapa de tradução. A versão produzida foi comparada com a escala original para melhoria do controle de qualidade. A etapa de adaptação transcultural foi composta por análise de conteúdo da escala por um comitê de especialistas e um pré-teste envolvendo 12 pacientes e 12 indivíduos saudáveis. A composição do comitê de especialistas seguiu as diretrizes de Beaton et al. ${ }^{8}$, sendo constituído por quatro fisioterapeutas, um educador físico, três médicos, dois brasileiros ex-universitários americanos, um tradutor nativo da língua portuguesa e um retrotradutor nativo da língua inglesa. O comitê foi orientado a ler atentamente as duas versões e sugerir modificações e correções, explicitando a razão, com a intenção de melhorar a adaptação ao contexto brasileiro, visando manter uma linguagem simples, melhorar a compreensão dos itens e aumentar sua clareza. Dessa forma, foi cumprida a etapa de equivalência semântica, idiomática, experimental e conceitual. Uma nova versão corrigida pôde ser produzida, considerando as modificações propostas. Uma vez que não existe método estatístico para avaliar o conteúdo de um questionário, essa etapa dependeu essencialmente do consenso entre especialistas, não havendo a necessidade de compará-lo a qualquer outro instrumento, conforme proposto por Cronbach \& Meehl 10. A versão final da escala foi submetida a uma profissional especializada em língua portuguesa para revisão.

Um pré-teste foi realizado com o objetivo de identificar possíveis dificuldades de compreensão derivadas da forma de aplicação, da forma de apresentação, do conteúdo das questões e das alternativas de respostas. Pacientes com diagnóstico de esclerose múltipla de acordo com os critérios de McDonald et al. 11, que se encontravam em acompanhamento no HUGG, foram incluídos consecutivamente. O grupo controle, selecionado no setor de fisioterapia do HUGG, foi formado por profissionais e acadêmicos com características demográficas semelhantes aos pacientes com esclerose múltipla. Após a leitura de cada item da MSWS-12, foram questionados se haviam entendido a pergunta. O resumo das etapas do processo de tradução e adaptação transcultural está apresentado na Tabela 1. Por fim, a medida de consistência interna do instrumento foi obtida pelo coeficiente alfa de Cronbach.

\section{Resultados}

A etapa de tradução produziu quatro versões independentes; após a reconciliação foi elaborada uma versão única. A questão “Made standing

Tabela 1

Resumo das etapas do processo de tradução e adaptação transcultural.

\begin{tabular}{|c|c|c|}
\hline Tradução & Objetivos & Resultados \\
\hline Etapa 1: Preparação & $\begin{array}{l}\text { Obter permissão para utilização do instrumento; } \\
\text { recrutar pessoal para participar do processo }\end{array}$ & $\begin{array}{l}\text { Autorização do autor; seleção de tradutores, } \\
\text { retrotradutores, especialistas, pacientes e controles }\end{array}$ \\
\hline Etapa 2: Tradução para a língua portuguesa & $\begin{array}{l}\text { Desenvolver versões independentes; fornecer } \\
\text { suporte teórico sobre os conceitos envolvidos } \\
\text { no instrumento }\end{array}$ & $\begin{array}{c}\text { Quatro versões independentes de dois tradutores } \\
\text { nativos na língua inglesa e dois nativos na língua } \\
\text { portuguesa }\end{array}$ \\
\hline Etapa 3: Reconciliação & Reconciliar as versões em uma versão única & Versão traduzida \\
\hline Etapa 4: Retrotradução & $\begin{array}{l}\text { Demonstrar a qualidade da tradução de } \\
\text { significados semelhantes da língua fonte }\end{array}$ & $\begin{array}{l}\text { Duas versões independentes de tradutores nativos } \\
\text { na língua inglesa }\end{array}$ \\
\hline Etapa 5: Revisão da retrotradução & Assegurar a equivalência conceitual da tradução & $\begin{array}{c}\text { Identificação de discrepâncias em relação à versão } \\
\text { original }\end{array}$ \\
\hline Etapa 6: Harmonização & $\begin{array}{c}\text { Compartilhar soluções para eventuais } \\
\text { discrepâncias conceituais }\end{array}$ & Versão única pela retrotradução \\
\hline
\end{tabular}

(continua) 
Tabela 1 (continuação)

\begin{tabular}{|c|c|c|}
\hline Tradução & Objetivos & Resultados \\
\hline Etapa 7: Adaptação transcultural & $\begin{array}{l}\text { Avaliar o nível de compreensão e equivalência } \\
\text { cognitiva; testar alternativas para a tradução }\end{array}$ & $\begin{array}{l}\text { Sugestões oriundas de } 12 \text { especialistas, } 12 \\
\text { pacientes e } 12 \text { indivíduos saudáveis }\end{array}$ \\
\hline $\begin{array}{l}\text { Etapa 8: Revisão do resultado da adaptação } \\
\text { transcultural e finalização }\end{array}$ & $\begin{array}{c}\text { Incorporar achados do processo de } \\
\text { interrogatório para melhoria do processo de } \\
\text { tradução }\end{array}$ & Modificações necessárias nas questões e respostas \\
\hline Etapa 9: Correção do texto revisado & Conferir possíveis erros do processo de tradução & $\begin{array}{l}\text { Revisão realizada por um profissional especializado } \\
\text { em língua portuguesa }\end{array}$ \\
\hline Etapa 10: Relatório final & $\begin{array}{l}\text { Descrever a metodologia utilizada e decisões } \\
\text { tomadas no processo de tradução }\end{array}$ & Versão final \\
\hline
\end{tabular}

when doing things more difficult?" necessitou de maior esforço no ajuste da tradução e da retrotradução. As outras questões apresentaram traduções semelhantes.

A fim de harmonizar a tradução, foi elaborada uma nova versão para a questão: "Dificultou ficar em pé ao fazer coisas?”. Em seguida a questão foi reenviada aos retrotradutores com resultado semelhante à escala original. A questão "Affected how smoothly you walk?" foi retrotraduzida de forma diferente da escala original, porém com concordância entre os retrotradutores. Neste caso, os ajustes foram feitos pelo comitê de especialistas, uma vez que foi necessário realizar a equivalência idiomática.

Após analisar as sugestões e comentários do comitê de especialistas, foi obtido consenso em diversas questões. Nos casos em que a equivalência conceitual permitiu, foi modificado "limitou" por "dificultou”, assim como "caminhar" por "andar" e "pode" por "consegue". Duas questões apresentaram modificações mais significativas: "Made standing when doing things more difficult?" para "Dificultou fazer as coisas em pé?" e "Affected how smoothly you walk?" para "Afetou o seu modo ou forma de andar?". As modificações foram necessárias para atender ao equivalente idiomático. Nesses casos, o significado geral transcendeu a literalidade, pois se considerou também o nível socioeconômico da população alvo da utilização da escala. O resultado final do processo de tradução e adaptação transcultural da versão brasileira da MSWS-12 (MSWS-12 BR) é apresentado na Figura 1.

Todos os pacientes e indivíduos saudáveis entenderam todas as questões (100\% "entenderam o que foi perguntado"), confirmando que a equivalência cultural da MSWS-12 BR foi realizada com sucesso. O tempo máximo para seu preenchimento foi menor que três minutos (171 segundos), mostrando sua aplicabilidade na prática (Tabela 2).
Por fim, a MSWS-12 BR apresentou um elevado valor de confiabilidade (alfa de Cronbach = $0,94)$, caracterizando a manutenção de sua consistência interna.

\section{Discussão}

As 10 etapas descritas nas diretrizes propostas por Wild et al. ${ }^{9}$ e Beaton et al. 8 foram integralmente cumpridas no presente estudo. No nosso melhor conhecimento, esse foi o primerio trabalho que realizou o processo de tradução e adaptação transcultural da MSWS-12 para a língua portuguesa no Brasil, não havendo possibilidade de comparação com estudos prévios.

Um estudo de adaptação transcultural cuidadoso faz parte de um adequado controle de qualidade da informação, que deve estar presente tanto em estudos epidemiológicos 12 como na prática clínica. Mucci et al. 13 e Conti et al. 14 demonstraram equivalência semântica sem a necessidade de mudanças da versão original. Já outros estudos 15,16 realizaram algumas adaptações metodológicas, assim como no presente estudo. Durante todo o processo de equivalência semântica, idiomática e conceitual, foi enfatizada a influência do nível sociocultural e da escolaridade na compreensão das questões pelos entrevistados. Em algumas questões, houve a sugestão de inserir um termo equivalente, o que é próprio de estudos desta natureza.

A MSWS-12 é constituida de ítens relacionados diretamente com a marcha e itens relacionados a equilíbrio, concentração e corrida, além de tarefas como subir e descer escadas. A combinação desses ítens representa uma forma mais abrangente de avaliação da mobilidade do indivíduo. A consistência interna encontrada no presente estudo $(0,94)$ foi próxima à encontrada em estudo prévio por Motl \& Snook $6(0,97)$. 
Versão brasileira da Multiple Sclerosis Walking Scale - 12 (MSWS-12 BR).

Estas perguntas são sobre sua dificuldade para andar nas duas últimas semanas devido à esclerose múltipla.

Para cada pergunta, por favor, marque o número que melhor descreve o seu grau de dificuldade.

Por favor, responda a todas as questões, mesmo que algumas delas pareçam semelhantes às outras, ou pareçam irrelevantes para você.

Se você não consegue andar de maneira alguma, por favor, marque aqui

Nas duas últimas semanas, o quanto a esclerose múltipla:

1. Limitou a sua capacidade de andar?

2. Limitou a sua capacidade de correr?

3. Limitou a sua capacidade de subir e descer escadas?

4. Dificultou fazer as coisas em pé?

5. Limitou o seu equilíbrio quando estava em pé ou andando?

6. Limitou a distância que você é capaz de andar?

7. Aumentou o esforço necessário para você andar?

8. Fez você utilizar apoio para andar dentro de casa (por exemplo: segurar em móveis, usar bengala etc.)?

9. Fez você utilizar apoio para andar fora de casa (por exemplo: usar bengala, andador etc.)?

10. Reduziu a velocidade de seu andar?

11. Afetou o seu modo ou forma de andar?

12. Fez que você precisasse se concentrar ou prestar atenção para andar?

Por favor, confira se você circulou UM número para CADA questão

1
1
1

$\begin{array}{cccc}\text { Em } & \text { Um } & \text { Moderadamente } & \text { Bastante }\end{array}$

2

2

2

$\begin{array}{ll}1 & 2 \\ 1 & 2\end{array}$

$\begin{array}{ll}1 & 2 \\ 1 & 2 \\ 1 & 2\end{array}$

$\begin{array}{ll}1 & 2 \\ 1 & 2 \\ 1 & 2 \\ 1 & 2\end{array}$

$+2$

3
3
3

2

4

5

$4-5$

4
5

5


Tabela 2

Características demográficas e clínicas de 12 pacientes com esclerose múltipla e 12 controles.

\begin{tabular}{lcc}
\hline & $\begin{array}{c}\text { Pacientes com esclerose múltipla } \\
\text { (média e desvio-padrão) }\end{array}$ & $\begin{array}{c}\text { Controles } \\
\text { (média e desvio-padrão) }\end{array}$ \\
\hline Idade & $33,92( \pm 6,80)$ & $33,17( \pm 7,28)$ \\
Sexo & 9 mulheres/3 homens & 9 mulheres/3 homens \\
Anos de estudo & $17,83( \pm 2,12)$ & $19,50( \pm 1,57)$ \\
IMC & $24,13( \pm 3,26)$ & $23,50( \pm 3,57)$ \\
MSWS-12 BR & $11,67( \pm 11,98)$ & $0,00( \pm 0,00)$ \\
Tempo de preenchimento & $88,75( \pm 39,03)$ & $48,44( \pm 26,30)$ \\
MFISTotal & $23,58( \pm 15,62)$ & $6,83( \pm 9,12)$ \\
MFISPhys & $11,50( \pm 6,76)$ & $3,58( \pm 5,05)$ \\
Velocidade da marcha & $1,25( \pm 0,20)$ & $1,20( \pm 0,19)$ \\
Cadência & $115,30( \pm 11,68)$ & $114,43( \pm 7,72)$ \\
EDSS & $1,22( \pm 1,20)$ & $\mathrm{NA}$ \\
Tempo de doença & $5,25( \pm 3,45)$ & $\mathrm{NA}$ \\
\hline
\end{tabular}

EDSS: Expanded Disability Status Scale; IMC: índice de massa corporal; MFIS: Modified Fatigue Impact Scale; MSWS-12 BR: versão brasileira da Multiple Sclerosis Walking Scale; NA: não aplicável.

\section{Conclusão}

As etapas de validação de conteúdo e consistência interna foram finalizadas de maneira satisfatória, mostrando que a MSWS-12 BR manteve as características originalmente descritas por Hobart et al. 5. Estudos futuros com uma população mais abrangente devem ser conduzidos para concluir o processo de validação da escala. 


\section{Resumo}

O baixo desempenho da caminhada é preditivo de doença cardíaca, de osteoporose e aumenta o risco de morte em idosos. A marcha associada à visão foram as funções corporais mais valiosas na percepção de pacientes com Esclerose Múltipla. O objetivo deste estudo foi realizar a tradução e a adaptação transcultural da Multiple Sclerosis Walking Scale - 12 (MSWS-12) para a língua portuguesa no Brasil. Um estudo de adaptação transcultural foi conduzido em dez etapas. Participaram do estudo quatro tradutores, dois retrotradutores, 12 especialistas, 12 pacientes, 12 indivíduos saudáveis e uma profissional especializada em língua portuguesa. Apenas a questão referente a fazer coisas em pé apresentou dificuldade no processo de tradução. O tempo máximo de preenchimento foi de menos de três minutos (171 segundos). A análise da consistência interna revelou um elevado valor de confiabilidade (alfa de Cronbach =0,94). As etapas de validação de conteúdo e consistência interna foram finalizadas de maneira satisfatória.

Marcha; Esclerose Múltipla; Tradução; Questionários

\section{Colaboradores}

L. A. C. Nogueira contribuiu com a concepção e delineamento do estudo, com a coleta, análise e interpretação dos dados e elaboração do texto. C. Baitelli participou da coleta, análise e interpretação dos dados e elaboração do texto. R. M. P. Alvarenga e L. C. S. Thuler contribuíram com a concepção e delineamento do estudo, interpretação dos dados e elaboração do texto.

\section{Agradecimentos}

À Coordenação de Aperfeiçoamento de Pessoal de Nível Superior (CAPES) pelo suporte financeiro.

\section{Referências}

1. Newman AB, Simonsick EM, Naydeck BL, Boudreau RM, Kritchevsky SB, Nevitt MC, et al. Association of long-distance corridor walk performance with mortality, cardiovascular disease, mobility limitation, and disability. JAMA 2006; 295:2018-26.

2. Harris J, Eng J. Goal priorities identified by individuals with chronic stroke: implications for rehabilitation professionals. Physiother Can 2004; 56:171-6.
3. Nogueira LA, Nobrega FR, Lopes KN, Thuler LC, Alvarenga RM. The effect of functional limitations and fatigue on the quality of life in people with multiple sclerosis. Arq Neuropsiquiatr 2009; 67:812-7.

4. Heesen C, Bohm J, Reich C, Kasper J, Goebel M, Gold SM. Patient perception of bodily functions in multiple sclerosis: gait and visual function are the most valuable. Mult Scler 2008; 14:988-91. 
5. Hobart JC, Riazi A, Lamping DL, Fitzpatrick R, Thompson AJ. Measuring the impact of MS on walking ability: the 12-Item MS Walking Scale (MSWS-12). Neurology 2003; 60:31-6.

6. Motl RW, Snook EM. Confirmation and extension of the validity of the Multiple Sclerosis Walking Scale-12 (MSWS-12). J Neurol Sci 2008; 268:69-73.

7. Guillemin F, Bombardier C, Beaton D. Cross-cultural adaptation of health-related quality of life measures: literature review and proposed guidelines. J Clin Epidemiol 1993; 46:1417-32.

8. Beaton DE, Bombardier C, Guillemin F, Ferraz MB. Guidelines for the process of cross-cultural adaptation of self-report measures. Spine (Phila Pa 1976) 2000; 25:3186-91.

9. Wild D, Grove A, Martin M, Eremenco S, McElroy S, Verjee-Lorenz A, et al. Principles of good practice for the translation and cultural adaptation process for patient-reported outcomes (pro) measures: report of the ISPOR Task Force for Translation and Cultural Adaptation. Value Health 2005; 8:94-104.

10. Cronbach LJ, Meehl PE. Construct validity in psychological tests. Psychol Bull 1955; 52:281-302.

11. McDonald WI, Compston A, Edan G, Goodkin D, Hartung HP, Lublin FD, et al. Recommended diagnostic criteria for multiple sclerosis: guidelines from the International Panel on the Diagnosis of Multiple Sclerosis. Ann Neurol 2001; 50:121-7.
12. Reichenheim ME, Moraes CL. Operationalizing the cross-cultural adaptation of epidemiological measurement instruments. Rev Saúde Pública 2007; 41:665-73.

13. Mucci S, Citero VA, Gonzalez AM, De Marco MA, Nogueira-Martins LA. Adaptação cultural do Chronic Liver Disease Questionnaire (CLDQ) para população brasileira. Cad Saúde Pública 2010; 26:199-205.

14. Conti MA, Latorre MR, Hearst N, Segurado A. Fatores associados à atividade física, comportamento sedentário e participação na Educação Física em estudantes do Ensino Médio em Santa Catarina, Brasil. Cad Saúde Pública 2009; 25:2179-86.

15. Lima DA, Lourenco RA. Adaptação para o Português da seção A do Cambridge Examination for Mental Disorders of the Elderly - Revised Version (CAMDEX-R) para o diagnóstico de demência. Cad Saúde Pública 2010; 26:1345-54.

16. Silva FC, Thuler LC. Cross-cultural adaptation and translation of two pain assessment tools in children and adolescents. J Pediatr (Rio J.) 2008; 84:344-9.

Recebido em 10/Jun/2011

Versão final reapresentada em 05/Fev/2012

Aprovado em 13/Fev/2012 